ISSN 2447-9071

doi https://doi.org/10.36414/rbmc.v7i17.89

Contato para correspondência: Leonardo Luiz Borges

E-mail:

leonardo.cbb@pucgoias.edu.br

Conflito de interesse: Não

Financiamento: Recursos próprios

Recebido: 01/04/2021

Aprovado: 22/04/2021

\section{Busca in silico de moléculas com potencial antitumoral a partir da espécie Aloysia citriodora}

\section{In silico search for molecules with antitumor potential from the species Aloysia citriodora}

\author{
Gabriel Diniz Mello', João Pedro Soares Dantas', Leonardo Luiz Borges ${ }^{1,2}$ \\ ${ }^{1}$ Pontifícia Universidade Católica de Goiás - PUC Goiás \\ ${ }^{2}$ Universidade Estadual de Goiás - UEG
}

\begin{abstract}
Resumo
Aloysiacitriodora Palau, conhecida como "salva-limão",éuma plantamedicinal popularmenteutilizada por seus potenciais antimicrobianos, neuroprotetores, antitumorais, entre outros. Tais potenciais são possiveis devido aos diversos quimiotipos, que apresentam substâncias bioativas, presentes na composição do óleo essencial da planta. O objetivo deste estudo foi investigar moléculas com potencial antineoplásico empregando ferramentas in silico presentes na espécie A. citriodora. A partir desses compostos, realizou-se a predição farmacológica de cada um, a fim deencontraroefeito antineoplásico mais promissor e seu respectivo receptor celular. Os métodos incluíram o uso de ferramentas in silico para avaliar atividades biológicas previstas. Também foram realizados estudos docagem molecular. Estudo concluique o composto neral apresentou o maior potencial para ligação molecularcom alvos tumorais, demonstrando uma potencial interação com a aldeído desidrogenase 1A1, macromolécula associada a alguns tipos de câncer, como o de pulmão.
\end{abstract}

Palavras-Chave: Aloysia citriodora; Neral; ALDH 1 A1; Câncer.

\begin{abstract}
Aloysia citriodora Palau, known as "lemon sage", is a medicinal plant popularly used for its potential antimicrobials, neuroprotectors, antitumor, among others. Such potentials are possible due to the various chemotypes, which present bioactive, present in the composition of the essential oil of the plant. This study aimed to investigate molecules with antineoplastic potential using in silico tools current in the species A. citriodora. From these compounds, a pharmacological prediction was made for each one to find the most promising antineoplastic effects. The methods included using in silico tools to assess predicted biological activities, pharmacodynamics, and toxicity. Docking analyses studies were also carried out. This study concludes that the neral compound had the most significant potential for molecular binding with tumor targets. Thus, demonstrating a potential interaction with aldehyde dehydrogenase 1A1, a macromolecule associated with some types of tumors, such as lung cancer. Keywords: Aloysia citriodora; Neral; ALDH 1 A1; Cancer.
\end{abstract}




\section{Introdução}

Conhecida popularmente por "salva-limão" ou "erva-lúcia", a espécie Aloysia citriodora Palau é nativa da América do Sul, sendo levada à África do Norte e Sul da Europa por portugueses e espanhóis do século XVII. Como planta medicinal cultivada ao redor do mundo, desempenhou papel de destaque na medicina da cultura Inca, e, atualmente, possui vasta utilização em escala industrial na condimentação de alimentos, gastronomia, agronomia, cosméticos e perfumaria.

Conforme a especificidade geográfica da região, a espécie vegetal aromática, da família das verbenáceas, pode apresentar variações na composição de seus óleos essenciais. Em relação ao teor terapêutico, estudos apontam que a espécie A. citriodora apresenta potencial antimicrobiano, neuroprotetor e antitumoral'. Tradicionalmente é utilizada para o alívio de sintomas gastrointestinais e para ansiedade.

Nesse sentido, tendo como base a composição do óleo essencial da planta, sabe-se da existência de diversos quimiotipos que apresentam substâncias bioativas1. Dentre os principais compostos biologicamente ativos da espécie (como terpenos, monoterpenos, quinonas, alcaloides e flavonoides), podemos citar as substâncias: citral, alfa-tujona, limoneno, carveol e neral. Esses compostos apresentam efeitos enzimáticos referentes à via oxidativa do metabolismo do álcool, a receptores dopaminérgicos, receptores muscarínicos, além de efeitos em hormônios sexuais e receptores associados a neoplasias².

Com o avanço da biologia molecular, bem como da bioquímica computacional, é possível predizer o efeito biológico de dada substância em relação à determinado receptor celular. Desse modo, tendo ciência dos efeitos farmacológicos provocados por substâncias naturais específicas, tem-se a possibilidade de encontrar potencial para obtenção de novos candidatos a fármacos ${ }^{3}$. Tal panorama compõe as recentes técnicas in silico para investigação deles, conhecendo-se a topografia tridimensional do receptor e seu sítio de interação molecular. Pode-se caracterizar, com o modelo de estudo in silico, propriedades e estrutura proteicas, bem como predizer suas singularidades físico-químicas, estabilidade e interação ligante-receptor.

As simulações biocomputacionais são uma alternativa acessível aos experimentos in vivo, vez que constituem uma ferramenta tecnológica contemporânea com aceitável poder de predição, além de contribuírem para a quebra de paradigmas histórico-científicos ${ }^{4}$.

O objetivo deste trabalho foi investigar o potencial antineoplásico do composto neral presente na espécie A. citriodora por meio de ferramentas in silico.

\section{Métodos}

Através de uma busca em artigos científicos nos servidores Science Direct, PubMed e Scielo, foi possível identificar os diversos quimiotipos bioativos da Aloysia citriodora Palau. Após a seleção dos compostos, foi realizada a codificação das moléculas para a realização das predições por meio do site Pubchem ${ }^{5}$. Realizou-se então a etapa de triagem de bioatividade dos compostos da planta, por meio dos programas SwissADME2, PASS 6 e SwisTargetPrediction (http://www.swisstargetprediction.ch/). A partir da triagem, foi possível obter uma lista de possíveis atividades biológicas para cada substância triada, de acordo com suas estruturas químicas, com base nas metodologias específicas de cada programa. Após a seleção dos compostos, foi realizada pesquisa de possíveis alvos para a verificação do docking molecular por meio do programa SuperPred Webserver, um servidor capaz de analisar o potencial de interação entre o alvo selecionado e o ligante investigado. Esses alvos foram obtidos no banco de dados Protein Data Bank?

Os compostos com maiores scores para a atividade antitumoral foram selecionados para simulações de docking molecular. O visualizador Hermes GOLD Suite 5.7.08 foi usado para preparar os receptores para o acoplamento na análise de redocking e os softwares Pymol e DS Viewer foram empregados para a geração das imagens com os resultados do docking molecular com o alvo selecionado. A região de interesse usada para o docking foi definida para a verificação das potenciais interações com o ligante de interesse. Valores padrão de todos os outros parâmetros foram utilizados e os complexos foram submetidos a corridas de algoritmos genéticos usando a função de score CHEMPLP'. Para validação dos parâmetros do modelo foi realizado o redocking utilizando o complexo liganteproteína cristalografada e essas condições foram utilizadas para a realização do docking com o ligante selecionado da espécie Aloysia citriodora.

\section{Resultados}

Entre o conjunto de compostos investigados na espécie Aloysia citriodora Palau, foram selecionados pelos critérios de Lipinski: neral, alfa e beta-thujone, limonene, carveol, sabinene, citral e gerarial. De acordo com as predições de propriedades farmacocinéticas, todos os compostos citados anteriormente foram classificados como druglikeness, conforme exposto na Tabela 1. 
Tabela 1. Conjunto de compostos presentes na espécie Aloysia citriodora e suas propriedades pelos critérios de Lipinski.

\begin{tabular}{cccccc}
\hline Composto & MM & Absorção Gl & $\begin{array}{c}\text { Permea- } \\
\text { bilidade à } \\
\text { BHE }\end{array}$ & Classe & Druglike \\
\hline Neral & 152,23 & Alta & Yes & Solúvel & Sim \\
Geraniol & 154,25 & Alta & Yes & Solúvel & Sim \\
alpha- & 152,23 & Alta & Yes & Solúvel & Sim \\
$\begin{array}{c}\text {-Tujona } \\
\text { beta-Tujona }\end{array}$ & 152,23 & Alta & Yes & Solúvel & Sim \\
Citral & 152,23 & Alta & Yes & Solúvel & Sim \\
$\begin{array}{c}\text { (-) - trans- } \\
\text {-Carveol } \\
\text { Sabinene }\end{array}$ & 152,23 & Alta & Yes & Solúvel & Sim \\
Limonene & 136,23 & Baixa & Yes & Solúvel & Sim \\
\hline
\end{tabular}

Abreviações: MM: massa molecular; Gl: gastrointestinal; BHE: Barreira Hematoencefálica.

A partir da análise de predição de toxicidade, verificou-se que os compostos selecionados apresentaram baixos níveis de toxicidade, o que, aliado ao perfil farmacocinético favorável, os classifica como substâncias promissoras para análises in vitro e in vivo.Além de possuírem resultados de predição farmacocinética favoráveis considerando a administração por via oral, a predição de atividade biológica revelou que o composto neral apresentou o maior valor de $\mathrm{Pa}$ (probabilidade do composto analisado ser ativo) associado a menores valores de Pi (probabilidade do composto analisado ser inativo), apresentado, portanto, maior potencial para interação molecular com alvos tumorais. Além disso, o composto neral apresenta alto potencial de absorção pelo trato gastrointestinal (TGI) e alta permeabilidade pela barreira hematoencefálica (BHE), segundo o programa Swiss $\mathrm{ADME}^{2}$. Ademais, na predição de alvos moleculares, o servidor SwissTargetPrediction sugere uma provável interação da molécula de neral com a enzima ALDH 1 A1 (Figura 1) (aldeído desidrogenase $1 \mathrm{~A} 1$, presente em especial no citosol de células-tronco, bem como em células pulmonares, renais e medulares ${ }^{10}$.

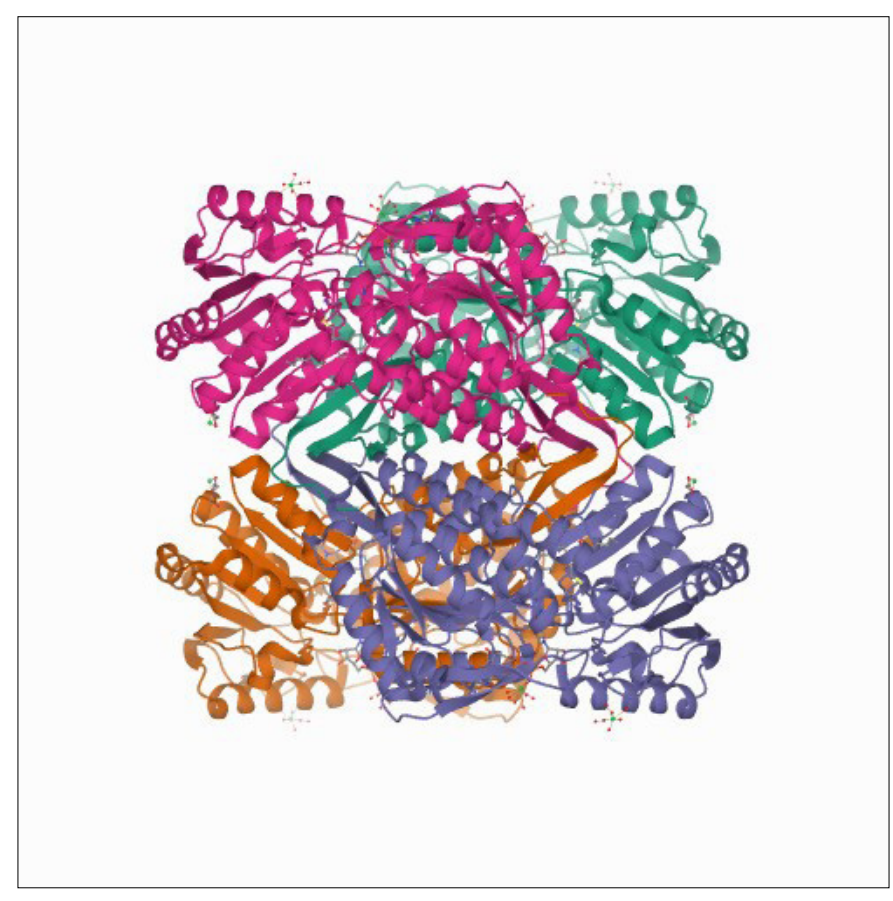

Figura 1. Estrutura tridimensional do alvo aldeído desidrogenase 1A1 (PDB ID: 5AC2). Fonte: Koch et al., 2015; https://www.rcsb.org/ structure/5AC2.

Para a validação dos modelos de interação obtidos por docking molecular, uma análise de redocking com o ligante co-cristalizado do alvo aldeído desidrogenase $1 \mathrm{~A} 1$ obtido no PDB foi realizada (Figura 2). A análise do redocking indicou bons resultados, com 9 poses com valores baixos de distância interatômica-RMSD.

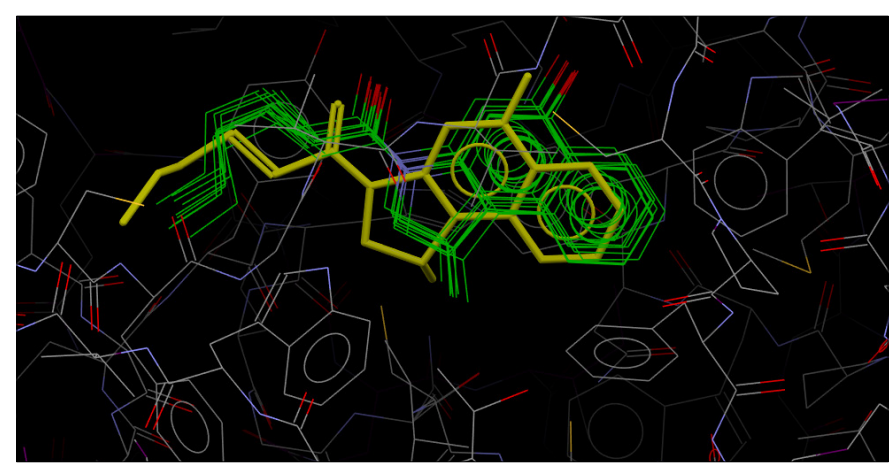

Figura 2. Redocking do ligante co-cristalizado (1-[(1S)-1-methyl5-oxidanyl-1,2-dihydrobenzo[e]indol-3-yl] hexan-1-one) no alvo ALDH $1 \mathrm{~A} 1$ (aldeído desidrogenase $1 \mathrm{~A} 1$ (os valores de RMSD das 9 poses geradas foram próximos a $1 A$ ).

Realizou-se então simulações de docking molecular empregando as mesmas condições do modelo do redocking para o 
alvo ALDH 1A1 (função de score: Chemscore). A Figura 3 traz a representação da interação entre o marcador químico neral e o sítio ativo do alvo selecionado. A partir dos resultados do docking molecular, é possível sugerir uma potencial interação entre o composto neral e o sítio catalítico do alvo aldeído desidrogenase (que guarda relação com alguns tipos de tumores). As principais interações foram com os resíduos de ASN121 (ligação de hidrogênio) e ligações $\pi$-alquila com PHE466, TRP178, CYS303, ILE304 e CYS302. A ligação de hidrogênio do neral com o resíduo de ASN131 constitui a interação intermolecular de maior intensidade e a maior responsável pela maior intensidade de ancoramento com a cavidade ativa da enzima ALDH 1A1. As demais interações sugeridas pela análise constituem interações de menor intensidade ( $\pi$-alquila), mas de importância no posicionamento do neral na cavidade ativa.
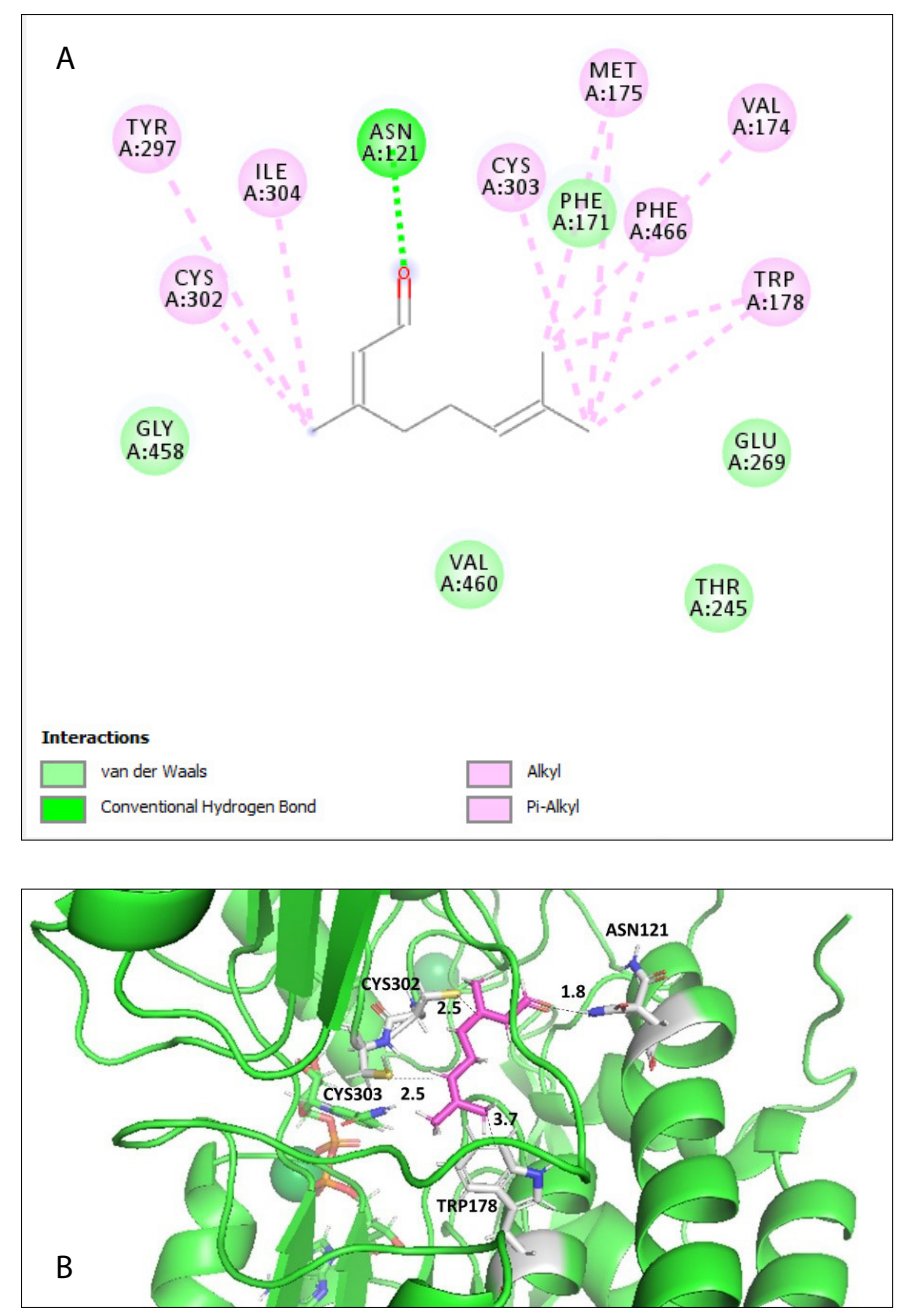

Figura 3. A-Diagrama 2D de interação do neral na posição 1 na enzima ALDH 1A1 Essa figura foi gerada com Discovery Studio 3.5Visualizer. B-Pose 1 da ancoragem do neral do sítio catalítico da enzima ALDH 1A1. Figura gerada com o software Pymol 1.1r1 software.

\section{Discussão}

Através da investigação e revisão dos metabólitos bioativos da espécie A. citriodora, foi possível selecionar o composto neral como objeto de estudo para as análises sobre os efeitos antitumorais atribuídos à planta. Pelo crivo da predição farmacocinética, $\mathrm{o}$ composto supracitado obteve os melhores resultados em termos de classificação druglikeness, toxicidade, bem como previsão da atividade biológica. Por meio da realização do docking molecular, observou-se que o neral possui a maior probabilidade de interação com a enzima ALDH $1 \mathrm{~A} 1$ (presente na maioria dos genomas de vertebrados, em especial no citosol de células-tronco, bem como em células pulmonares, renais e medulares humanas) ${ }^{11}$, a qual desempenha papel central nos mecanismos de transcrição no núcleo celular. A aldeído desidrogenase $1 \mathrm{~A} 1$ compõe uma família de outras 19 isoenzimas ${ }^{11}$.

Apesar de ser utilizada como marcador de células-tronco e células-tronco cancerígenas, o reconhecimento das funções específicas na engenharia celular de regulação da ALDH 1A1 em células-tronco permanece, ainda, obscuro12. Sabe-se que, do ponto de vista fisiológico, a enzima ALDH $1 \mathrm{~A} 1$ converte retinaldeído (produto do retinol) em ácido retinoico (AR). Oácido em questão, por sua vez, atua como ligante para receptores nucleares, e regula a atividade transcricional de genes associados à proliferação e diferenciação celulares. $O$ AR, portanto, pode interagir com os receptores nucleares RA e RX e ativar a expressão gênica relacionada à diversos fenômenos, dentre os quais a perda de marcadores celulares, interrupção do ciclo celular e alteração da morfologia da célula. Ocorre, nesse contexto, um processo de feedback positivo relacionado à regulação positiva desses receptores e a sinalização do retinoide, promovendo, assim, o crescimento tumoral e induzindo a carcinogênese em célulastronco neoplásicas ${ }^{12}$.

Além disso, algumas isoenzimas da ALDH, dentre elas a $1 \mathrm{~A} 1$, possuem evidências de desempenhar função de proteção contra agentes citotóxicos, dentre eles os quimioterápicos, além de oferecer resistência à radiação em células cancerosas ${ }^{12}$. Em termos clínicos, a deficiência da proteína está envolvida também com piora sintomatológica do Mal de Parkinson ${ }^{13}$, resistência insulínica/obesidade ${ }^{14}$ e maior sensibilidade ao álcool ${ }^{15}$. Em contrapartida, a isoenzima, quando em altos níveis ou superexpressão, pode indicar mau prognóstico de variados tipos de câncer, como câncer de mama ${ }^{10}$.

Este estudo sugere que o composto neral, encontrado nos óleos essenciais da espécie $A$. citriodora, pode interagir com a aldeído desidrogenase $1 \mathrm{~A} 1$ por meio de ligações de hidrogênio (ancoragem na cavidade ativa) e $\pi$-alquila (posicionamento no sítio catalítico). Dessa forma, tendo como base as forças de ligação moleculares, tal interação tem a capacidade de promover 
respostas biológicas de interesse na fisiologia carcinogênica, sendo as ligações de hidrogênio essenciais para a estabilidade da ligação entre os compostos neral e ALDH 1A1. Em uma pesquisa foi sugerido que o composto neral pode induzir a apoptose em células leucêmicas humanas e em células do câncer de mama. Esses mecanismos de ação podem estar associados a inibição de alvos essenciais à existência de células tumorais, como a aldeído desidrogenase $1 \mathrm{~A} 1^{16}$.

Portanto, deve-se considerar essencial o aprofundamento de investigações acerca do potencial do composto neral para células cancerígenas. Atualmente, diversos tipos de neoplasias malignas ameaçam a vida de milhões de pessoas em todo o mundo, o que significa dizer que a temática em análise envolve não somente questões científicas, mas também humanitárias.

\section{Conclusão}

As análises in silico sugerem que o composto neral mostrou-se o mais promissor para futuras investigações em testes in vitro e in vivo devido aos seus maiores scores encontrados nas ferramentas de predição e ao resultado in silico de sua interação com um alvo molecular associado a câncer. Além disso, este trabalho fornece subsídios para explicar evidências de propriedades biológicas da espécie Aloysia citriodora.

\section{Referências}

1. Bahramsoltani R, Rostamiasrabadi P, Shahpiri Z, Marques AM, Rahimi R, Farzaei MH, Aloysia citrodora Paláu (Lemon verbena): a review of phytochemistry and pharmacology, Journal of Ethnopharmacology. 2018;222(10):34-51.

2. Daina A, Michielin O, Zoete V. SwissADME: a free web tool to evaluate pharmacokinetics, drug-likeness, and medicinal chemistry friendliness of small molecules. Sci Rep. 2017;7(42717).

3. Poroikov VV, Filimonov DA. How to acquire new biological activities in old compounds by computer prediction. Journal of Computer-Aided Molecular Design. 2002;16(11):819-24.

4. Kunh TS. A estrutura das revoluções científicas. 3. ed. São Paulo: Perspectiva; 2000.

5. Kim S, Chen J, Cheng T, Gindulyte A, He J, He S, et al. PubChem 2019 update: improved access to chemical data. Nucleic Acid Res. 2019;47(D1):D1102-09.

6. Poroikov VV, Filimonov DA, Borodina YV, Lagunin AA, Kos A. Robustness of biological activity spectra predicting by computer program PASS for Noncongeneric Sets of Chemical Compounds. J. Chem. Inf. Comput. Sci. 2000;40(6):1349-55.

7. Berman HM, Westbrook J, Feng Z, Gilliland G, Bhat TN,
Weissig $\mathrm{H}$, et al. The Protein Data Bank. Nucleic Acid Res. 2000;28(1):235-42.

8. Cole JC, Nissink JWM, Taylor R. Protein-ligand Docking and Virtual Screening with GOLD in Virtual Screening in Drug Discovery. Shoichet B, Alvarez J, Eds.; Taylor \& Francis CRC Press. 2005.

9. Korb O, StützleT, ExnerTE. Empirical scoring functions for advanced protein-ligand docking with PLANTS. J Chem Inf Model. 2009;49(1):84-96.

10. Xu X, Chai S, Wang P, Zhang C, Yang Y, Yang Y, et al. Aldehyde dehydrogenases and cancer stem cells. Cancer Lett. 2015; 369:50-7.

11. King G, Holmes R. Human corneal and lens aldehyde dehydrogenases. Purification and properties of human lens ALDH1 and differential expression as major soluble proteins in human lens (ALDH1) and cornea (ALDH3). Adv Exp Med Biol. 1997;414:19-27.

12. Tomita H, Tanaka K, Tanaka T, Hara A. Aldehyde dehydrogenase $1 \mathrm{~A} 1$ in stem cells and cancer. Oncotarget. 2016;7:11018-32.

13. Basso M, Giraudo S, Corpillo D, Bergamasco B, Lopiano L, Fasano M. Proteome analysis of human substantia nigra in Parkinson's disease. Proteomics. 2004;4(12):3943-52.

14. Berry DC, Noy N. All-trans-retinoic acid represses obesity and insulin resistance by activating both peroxisome proliferation-activated receptor beta/delta and retinoic acid receptor. Mol Cell Biol. 2009;29(12):3286-96.

15. Bhave SV, Hoffman PL, Lassen N, Vasiliou V, Saba L, Deitrich RA, et al. Gene array profiles of alcohol and aldehyde metabolizing enzymes in brains of C57BL/6 and DBA/2 mice. Alcohol Clin Exp Res. 2006;30(10):1659-69.

16. De Martino L, D'Arena G, Minervini MM, Deaglio S, Fusco BM, Cascavilla N, et al. Verbena officinalis Essential Oil and its Component Citral as Apoptotic-Inducing Agent in Chronic Lymphocytic Leukemia. Int J Immunopathol Pharmacol. 2009;1097-1104. 